
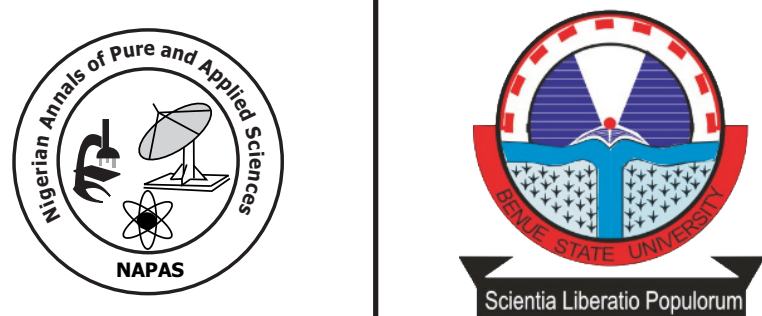

\title{
Comparison of Vegetable Oil-Silicone Oil Interfacial Tension Data from the du NoüyRing and the Spinning Drop Methods
}

\author{
Tyowua, A.T. ${ }^{1,{ }^{*}}$ Targema M. ${ }^{2}$ and Binks, B.P. ${ }^{1}$ \\ ${ }^{1}$ School of Mathematics and Physical Sciences, \\ University of Hull, Hull. HU6 7RX. UK \\ ${ }^{2}$ Applied Colloid Science and Cosmeceutical Group, \\ Department of Chemistry, \\ Benue State University, PMB 102119, Makurdi, Nigeria \\ "Corresponding author's atyowua@bsum.edu.ng/+2349091818649
}

\begin{abstract}
Liquid-liquid interfacial tension is very important in colloid and surface chemistry as it detects the amount of energy required to create a new interface. This makes its measurement very crucial. There are different techniques for measuring it, with the common ones being the du Noüy ring and spinning drop methods. The aim of this work is to compare the low interfacial tension values obtained from the du Noüy ring method with those obtained using the spinning drop method in vegetable oil-silicone oil systems. Low interfacial tension values of various vegetable oil-silicone oil systems were measured with the du Noüy ring and spinning drop methods. The interfacial tension values range from 2.1 to $2.8 \mathrm{~m} \mathrm{~N} \mathrm{~m}^{-1}$ for the du Noüy ring method and 1.0 to $2.7 \mathrm{mN} \mathrm{m}^{-1}$ for the spinning drop method. These values agree closely) $05.0(=p$. This shows that both methods can be used for measuring interfacial tension with high accuracy in vegetable oil-silicone oil systems.
\end{abstract}

Key words: surface tension, interface, interfacial tension, colloid chemistry, surface chemistry 


\section{Introduction}

Intermolecular forces are responsible for the surface tension of liquids (Sophocleous, 2010). These forces are of two types, namely the cohesive forces which represent the attractive forces between molecules of the same substance and the adhesive forces which represent the attractive forces between molecules of different substances (Sophocleous, 2010). The cohesive forces are responsible for the surface tension of liquids. The liquid molecules at the surface have less number of neighbours on all sides, compared with those in the bulk, and as a result cohere more strongly with the neighbouring molecules than those in the bulk (Hunter, 1989). This gives rise to a surface film which acts like an elastic membrane. The same thing happens at the interface of two immiscible liquid phases, resulting in the formation of interfacial tension (Hunter, 1989). Therefore, surface tension is the force acting per unit length on either sides of an imaginary line drawn on the free surface of a liquid, with the direction of action being perpendicular to the imaginary line and tangential to the free surface of the liquid (Tyowua, 2017). When the free surface is between two immiscible liquids, the term "interface" is used with the corresponding force being the interfacial tension. Strictly, surface tension is the tension of the liquid-air interface and it is sometimes called the liquid-air interfacial tension. The SI units of surface and interfacial tensions are $\mathrm{N} \mathrm{m}^{-1}$, although other units like dynes $\mathrm{cm}^{-1}$ and $\mathrm{J}$ $\mathrm{m}^{-2}$ are also known (Tyowua, 2017). Surface tension is responsible for many physical phenomena. For example, it is responsible for the spherical nature of gas bubbles and liquid drops (de Gennes et al., 2004). It is also responsible for capillarity, the rising or falling of liquids in thin tubes. Interfacial tension is very important in colloid science as it determines the amount of energy required to create a new interface as well as the size of the new interface created (Cosgrove, 2005).

The du Noüy ring and spinning drop methods are commonly used in the measurement of liquid-liquid interfacial tension. Other methodsare also available and can be found in the review of Drelich et al.,(2002). The du Noüy ring is said to measure interfacial tensions values down to $0.1 \mathrm{mN} \mathrm{m}^{-1}$ with limited accuracy at values $\leq 5$

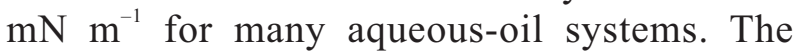
spinning drop method measures interfacial tension values down to $\geq 10^{-4} \mathrm{mN} \mathrm{m}^{-1}$ with high level of accuracy (Drelich et al., 2002). As a result, it is highly recommended for low interfacial tension measurement. Unfortunately, the spinning drop tensiometer is expensive, requires more liquid and is slower compared to the du Noüy ring method. The aim of this work is to compare the low interfacial tension values obtained from the du Noüy ring method with those obtained using the spinning drop method in vegetable oil-silicone oil systems.

\section{Materials and Methods Materials}

Three different vegetable oils and three different silicone oils of varying viscosity were used.The vegetable oilsweresunflower oil (Tesco), olive oil (Sigma) and rapeseed oil (Fluka) while the silicone oils were silicone oil 20,50 and $100 \mathrm{cS}$ (all from Dow Corning). The vegetable oils were passed twice through a column containing basic alumina to remove polar impurities before use while the silicone oils were used as received.

\section{Methods}

\section{Density Measurement}

The density of the oils was measured at 25 ${ }^{\circ} \mathrm{C}$ using the Anton Paar DMA 35N digital densitometer. Measurements were done in triplicate. The cleaning of the instrument between measurements, necessary for accurate values, consisted of washing with copious amounts of ethanol, followed by water and drying at ambient conditions. The instrument has a filling tube (inner diameter $0.2 \mathrm{~cm}$ and length $18 \mathrm{~cm}$ ) through which the liquid goes into it when the pressure applied at the pump lever is released. Filling the measuring cell with the oil $\left(\approx 2 \mathrm{~cm}^{3}\right)$ consisted of pressing down the pump lever as far as possible, submerging the filling tube in the sample and slowly releasing the pump lever.

\section{Surface Tension and Interfacial Tension Measurement Using the du NoüyRing}

TheKrüss K12 digital tensiometer using the $\mathrm{du}$ Noüy ring was used for the measurement. The detachment mode was used for the measurement of oil surface tension (relatively high) while the push mode was used for the measurement of vegetable oil-silicone oil interfacial tension (relatively low). In both cases, the measurements were done in triplicate. Prior to each measurement, the glass vial containing the sample was washed with $0.5 \mathrm{M}$ alcoholic $\mathrm{KOH}$ solution and then with Ultrapure water and dried at ambient conditions. In addition, the du Noüy ring was heated to glowing in a blue Bunsen flame.

In the detachment mode of the du Noüy ring method, surface tension valueis related to the maximum force $F$ required to detach the ring from the interface (Figure 1) (Harkins and Brown, 1919; Lecomte du Noüy, 1919). 


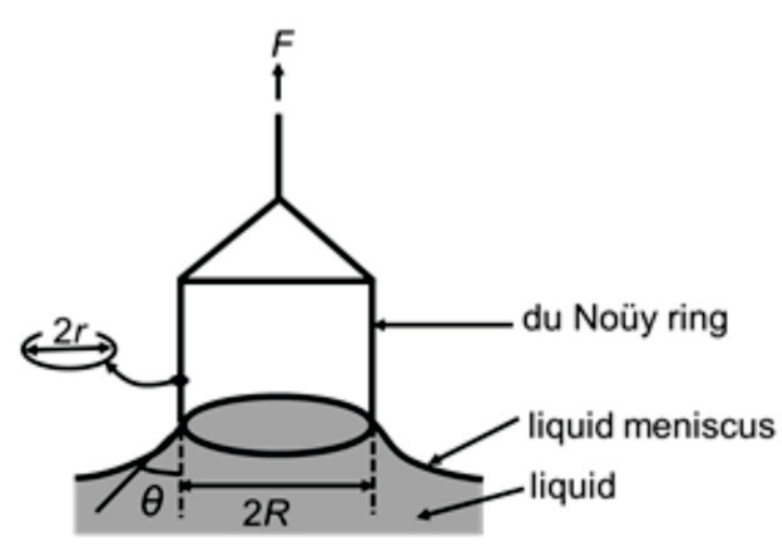

Figure 1. Schematics of the du Noüy ring showing the properties of the ring and how $F$ acts on it during a measurement in the detachment mode.

The ring is usually made of platinum or platinumiridium alloy of radius $R$ between $2-3 \mathrm{~cm}$. The radius $r$ of the wire usually ranges from $1 / 30$ to $1 / 60$ of that of the ring (i.e. $R / 30$ to $R / 60$ )(Vold and Vold, 1983). The surface tension value is calculated from Equation (1)(Lecomte du Noüy, 1919; Vold and Vold, 1983).

$\gamma_{\mathrm{la}}(\exp )=\frac{F}{P \cos \theta} f=\frac{F}{P} f=\frac{F}{4 \pi R} f$

In the detachment mode, $F$ is the force acting along the three-phase contact line and it is exactly equal to the weight of the liquid meniscus standing above the plane of the fluid interface. The $P$ is the perimeter of the three-phase contact line and it is equal to two times the circumference of the ring (i.e. $4 \pi R$ ). The $\theta$ stands for the contact angle between the liquid meniscus and the surface of the ring and it gives an idea of the surface wettability. The $\theta$ is necessarily required to be zero for accurate measurements. The $f$ is given by Equation (2) (Huh and Mason, 1975)

$f=\frac{\Delta \rho g R^{2}}{4 \pi \gamma_{\mathrm{la}}(\exp )}$

$f=\frac{\Delta \rho g R^{2}}{4 \pi \gamma_{\text {la }}(\exp )}$

Or Equation (3)(Zuidema and Waters, 1941)

$f=0.725+\left(\frac{3.63 \times 10^{-3} \gamma_{\mathrm{la}}(\exp )}{\pi^{2} \Delta \rho R^{2}}-\frac{1.679 r}{R}+0.04534\right)^{0.5}$

and it is a correction factor required to account for the additional volume of liquid lifted during the detachment of the ring from the surface and varies between 0.75 and 1.05. Finally, $\Delta \rho$ and $g$ are the density difference between theoil and air and gravitational acceleration, respectively.

From the foregoing, measured values of surface tension $\gamma_{\text {la }}(\exp )$ above $25 \mathrm{mN} \mathrm{m}^{-1}$ were corrected with the Huh and Mason,(1975) correction factor [Equation (2)] while those below $25 \mathrm{mN} \mathrm{m}^{-1}$ were corrected with the Zuidema and Waters,(1941) correction factor [Equation (3)]. The latter correction was done automatically by the instrument while the former correction was done manually in Microsoft Excel spread sheet.

In the push mode, surface tension is related to the maximum force required to break the interface. The surface tension values obtained here are not corrected as the liquid meniscus is not lifted up during the measurement process.

\section{Interfacial Tension Measurement Using the Spinning Drop Tensiometer}

Spinning drop tensiometry is based on the fact that gravity has little or no effect on the shape of a drop (less dense) suspended in the bulk of a liquid (more dense) contained in a horizontal capillary tube rotating about its horizontal axis where the two remain immiscible (Figure 2).

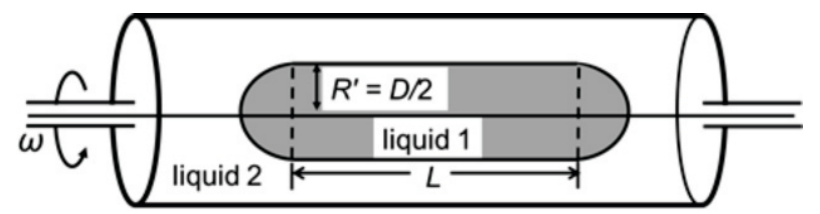

Figure 2. Schematic illustration of the spinning drop technique for measuring a liquid-liquid interfacial tension.

The drop is ellipsoidal at relatively low rotational speed $\omega$, but elongates and becomes cylindrical at relatively high $\omega$ where centrifugal forces dominate. Under the latter condition, the radius $R^{\prime}$ of the cylindrical drop is detected by the interfacial tension, difference in fluid density $\Delta \rho$ and $\omega$. As a result, the interfacial tension is calculated quite accurately from Equation (4) provided the ratio of the length $L$ of the cylindrical drop to its diameter $D$ is $\geq 4$ (Vonnegut, 1942).

liquid - liquid tension $=\frac{\Delta \rho \omega^{2} R^{3}}{4}\left(1+\frac{2 R^{\prime}}{3 L}\right)$

The vegetable oil (o)-silicone oil (o') interfacial tension'oorwas measured using the Krüss spinning drop tensiometer at $25^{\circ} \mathrm{C}$. A drop of a vegetable oil (less dense) was spun in the bulk of a silicone oil (more dense) at very high $\omega(\approx$ $8000 \mathrm{rpm}$ ) to obtain a cylindrical vegetable oil drop. The $D$ and $L$ of the vegetable oil drop were measured and used for calculating the oo $\gamma$ by using Equation (5) 
$\gamma_{\mathrm{oo}^{\prime}}=N^{2} D^{3} A \Delta \rho\left(\frac{n_{T}}{n_{\mathrm{o}^{\prime}}}\right)^{3}\left[1+\left(\frac{n_{T} D}{n_{\mathrm{o}^{\prime}}}\right) / 3 L\right]$

which has been obtained from Equation (4). The symbols $n_{T}=1.401$ (at $25^{\circ} \mathrm{C}$ ), ' $n$ o, $N$ and $A \approx 1.572$ $\times 10^{-8}$ represent the refractive index of the thermostating oil (silicone oil, Avocado Chemicals Ltd. UK), refractive index of the silicone oils, rotation speed in revolutions per min (cf. $\omega /$ radians per second) and all sample invariant conversion factors, respectively. The difference in silicone oil-vegetable oil density $(\Delta \rho)$ was obtained from the density measurement (given in Table 1). The was measured at $25^{\circ} \mathrm{C}$ using an Abbe refractometer (Hilger, England). The average of three separate measurements was $1.400,1.402$ and 1.403 for silicone oil 20, 50 and
$100 \mathrm{cS}$, respectively. Each vegetable oil-silicone oil interfacial tension was measured in triplicate.

\section{Statistical Analysis}

All measurements were done in triplicate. The results are expressed as mean values \pm standard deviation. The differences between the values of the du Noüy ring and spinning drop methodswere analysed using paired samples $t$-test at $95 \%$ confidence level.This was done with IBM SPSS Statistics programmeversion 20(IBM Corp., Armonk, NY, USA)for Windows.

\section{Results and Discussion}

\section{Density of Oils}

The various densities of the vegetable oils and silicone oils are given in Table 1.

Table 1. The average density (at $25^{\circ} \mathrm{C}$ ) of the vegetable oils and the silicone oils used. The values are quoted to $\pm 0.01 \mathrm{gcm}^{-3}$.

\begin{tabular}{ll}
\hline Oil & $\rho / \pm 0.01 \mathrm{gcm}^{-3}$ \\
\hline Sunflower oil & 0.92 \\
Olive oil & 0.91 \\
Rapeseed oil & 0.92 \\
Silicone oil $20 \mathrm{cS}$ & 0.95 \\
Silicone oil $50 \mathrm{cS}$ & 0.96 \\
Silicone oil $100 \mathrm{cS}$ & 0.97 \\
\hline
\end{tabular}

The results in Table 1 reveal that the density values for the silicone oils are higher than those of the vegetable oils. This indicates that the silicone oils are denser than the vegetable oils, even though their values are similar. Furthermore, the vegetable oils have similar density values, likewise the silicone oils. These values are in good agreement with those reported by Binks et al.,(2011).

\section{Surface Tension of Vegetable Oils and Silicone Oils}

Table 2 gives the surface tension of the vegetable oils and the silicone oils at $25^{\circ} \mathrm{C}$. It can be seen that the surface tension values of the vegetable oils are higher than those of the silicone oils. These values indicate that vegetable oils have stronger cohesive forces than the silicone oils. For the vegetable oils, the surface tension values agree closely with those reported by Michalski et al.,(1998). In a similar way, the surface tension values of the silicone oils corroborate with those reported in our previous work (Binks and Tyowua, 2013). The vegetable oils have similar surface tension values just like the silicone oils. Moreover, the surface tension values of the vegetable oils are higher than those of the silicone oils. These values indicate that the vegetable oils have stronger cohesive forces than the silicone oils.

Table 2. The average surface tension of the vegetable oils and the silicone oils at $25^{\circ} \mathrm{C}$. The values are quoted to $\pm 0.1 \mathrm{mN} \mathrm{m}^{-1}$.

\begin{tabular}{lc} 
Oil & Tensions/ $\pm 0.1 \mathrm{mN} \mathrm{m}^{-1}$ \\
Sunflower oil & 32.2 \\
Olive oil & 33.1 \\
Rapeseed oil & 33.8 \\
Silicone oil $20 \mathrm{cS}$ & 20.8 \\
Silicone oil $50 \mathrm{cS}$ & 21.2 \\
Silicone oil $100 \mathrm{cS}$ & 21.4 \\
\hline
\end{tabular}

Vegetable Oil-Silicone Oil Interfacial Tension

The vegetable oil-silicone oil interfacial tension values from the du Noüy ring and the spinning drop methods are presented in Table 3. 
Table 3. Averagevalues of vegetable oil-silicone oil interfacial tension ${ }^{\circ} \circ\left(25^{\circ} \mathrm{C}\right)$ from the (a) du Noüy ring and (b) spinning drop methods. The values are quoted to \pm 0.1 or $0.2 \mathrm{mN} \mathrm{m}^{-1}$. Values marked with asterisk (in the same row) are significantly different $) 05.0$ ( $=p$ from each other.

\begin{tabular}{lll}
\hline & \multicolumn{2}{c}{$\gamma_{\mathrm{oo}^{\circ}} / \mathrm{mN} \mathrm{m}^{-1}\left(\right.$ at $\left.25^{\circ} \mathrm{C}\right)$} \\
\cline { 2 - 2 } Vegetable oil-silicone oil system & $(\mathrm{a}) / \pm 0.1 \mathrm{mN} \mathrm{m}^{-1}$ & $(\mathrm{~b}) / \pm 0.2 \mathrm{mN} \mathrm{m}^{-1}$ \\
\hline Sunflower oil-silicone oil $20 \mathrm{cS}$ & 2.3 & 2.7 \\
Sunflower oil-silicone oil $50 \mathrm{cS}$ & 2.8 & 2.7 \\
Sunflower oil-silicone oil $100 \mathrm{cS}$ & $2.1^{*}$ & $1.0^{*}$ \\
Olive oil-silicone oil $20 \mathrm{cS}$ & 2.6 & 2.0 \\
Olive oil-silicone oil $50 \mathrm{cS}$ & 2.7 & 2.8 \\
Olive oil-silicone oil $100 \mathrm{cS}$ & $2.3^{*}$ & $1.0^{*}$ \\
Rapeseed oil-silicone oil $20 \mathrm{cS}$ & 2.7 & 2.3 \\
Rapeseed oil-silicone oil $50 \mathrm{cS}$ & 2.8 & 2.7 \\
Rapeseed oil-silicone oil $100 \mathrm{cS}$ & &
\end{tabular}

It can be seen that values from both methods are generally less than $3 \mathrm{mN} \mathrm{m}^{-1}$ and that the values increase with increasing viscosity of the silicone oils, i.e. going from 20 to $100 \mathrm{cS}$.In addition, values from both methods agree closely $) 05.0(=p$, except for the olive oil-silicone oil 20 cSand rapeseed oil-silicone oil $20 \mathrm{cSwhere}$ the values are significantly different. This is probably due to the fact that the silicone oil $20 \mathrm{cSof}$ lower viscosity allows the elongation of the vegetable oils more than necessary so that the ratio $D / L$ in Equation (5) is small, resulting in an overall low interfacial tension upon calculation. Suffice it to say that both methods can be used for reliable measurement of vegetable oil-silicone oil interfacial tension.

\section{Conclusions}

The du Noüy ringand the spinning drop methods are commonly used in the measurement of liquid-liquid interfacial tension. The du Noüy ring method measures low interfacial tensions with limited accuracy at values $\leq 5 \mathrm{mN} \mathrm{m}^{-1}$ for many aqueous-oil systems while the spinning drop method measures interfacial tension values down to $\geq 10^{-4} \mathrm{mN} \mathrm{m}^{-1}$ with high level of accuracy. This work shows that in vegetable oil-silicone oil systems, of low interfacial tensions $\left(<3 \mathrm{mN} \mathrm{m}^{-1}\right)$, the du Noüy ringand spinning drop methods give interfacial tension values that agree closely) 05.0 ( $=p$. This indicates that the du Noüy ringmethod, which is faster, less expensive and uses less liquid, may equally be used for such systems in the absence of a spinning drop tensiometer.

\section{References}

Binks, B.P. and Tyowua, A.T. (2013). Influence of the degree of fluorination on the behaviour of silica particles at air-oil surfaces. Soft Matter, 9(3): 834-845.

Binks, B.P., Rocher, A. and Kirkland, M. (2011). Oil foams stabilised solely by particles. Soft Matter, 7(5): 1800-1808.

Cosgrove, T., Ed. (2005). Colloid Science: Principles, Methods and Applications. Oxford, Blackwell Publishing.

deGennes, P.-G., Brochard-Wyart, F. and Quéré,
D. (2004). Capillarity and Wetting Phenomena. New York, Springer-Verlag.

Drelich, J., Fang, C. and White, C.L. (2002). Measurement of interfacial tension in fluidfluid systems. Encyclopedia of Surface and Colloid Science. New York, Marcel Dekker, Inc.:3152-3166.

Harkins, W.D. and Brown, F.E. (1919). The determination of surface tension (free surface energy), and the weight of falling drops: the surface tension of water and benzene by the capillary height method. $J$. Am. Chem.Soc., 41: 499-525.

Huh, C. and Mason, S.G. (1975). A rigorous theory of ring tensiometry. Colloid Polymer Sci., 253(7): 566-580.

Hunter, R.J. (1989). Foundations of Colloid Science. Oxford, Oxford University Press.

Lecomte du Noüy, P. (1919). A new apparatus for measuring surface tension. J. Gen. Physiol., $1: 521-524$.

Michalski, M.-C., Desobry, S., Pons, M.-N. and Hardy, J. (1998). Adhesion of edible oils to food contact surfaces. J. Amer. Oil Chem. Soc., 75(4): 447-454.

Sophocleous, M. (2010). Understanding and explaining surface tension and capillarity: an introduction to fundamental physics for water professionals. Hydrogeol. J., 18: 811821.

Tyowua, A.T. (2017). Modern Principles of Colloid and Surface Chemistry. Lagos, Nigeria, Academy Press.

Vold, R.D. and Vold, M.J. (1983). Colloid and Interface Chemistry. London, AddisonWesley Publishing Co.

Vonnegut, B. (1942). Rotating Bubble Method for the Determination of Surface and Interfacial Tensions. Rev. Sci. Instr., 13(1): 6-9.

Zuidema, H. and Waters, G. (1941). Ring Method for Determination of Interfacial Tension. Ind. Eng. Chem. Anal. Ed., 13(5):312-313. 